\title{
Technè
}

La science au service de l'histoire de l'art et de la préservation des biens culturels

42 | 2015

Science et conservation

\section{Restauration et documentation à Rome dans les années 1830. Le cas des vases grecs}

Restoration and documentation in Rome in the 1830s. The case of Greek vases

\section{Marie-Amélie Bernard}

\section{OpenEdition}

Journals

Édition électronique

URL : http://journals.openedition.org/techne/6525

DOI : 10.4000/techne.6525

ISSN : 2534-5168

Éditeur

C2RMF

Édition imprimée

Date de publication : 1 décembre 2015

Pagination : 25-33

ISBN : 978-2-7118-6249-8

ISSN : $1254-7867$

Référence électronique

Marie-Amélie Bernard, «Restauration et documentation à Rome dans les années 1830. Le cas des vases grecs », Technè [En ligne], 42 | 2015, mis en ligne le 01 décembre 2015, consulté le 11 mars 2021. URL : http://journals.openedition.org/techne/6525; DOI : https://doi.org/10.4000/techne.6525

La revue Technè. La science au service de l'histoire de l'art et de la préservation des biens culturels est mise à disposition selon les termes de la Licence Creative Commons Attribution - Pas d'Utilisation Commerciale - Pas de Modification 4.0 International. 
Marie-Amélie Bernard

\section{Restauration et documentation à Rome dans les années 1830. Le cas des vases grecs}

Restoration and documentation in Rome in the 1830s. The case of Greek vases.

Résumé. Le peu de valeur des restaurations du passé et l'inexistence de la documentation les concernant relèvent d'un dogme qui, confronté aux résultats de la recherche, vacille fortement. Cet article voudrait contribuer à le mettre à bas en prenant l'exemple de restaurations de vases grecs en Italie, et plus particulièrement à Rome, dans les années 1830 et 1840. Une thèse en cours sur le marchand et restaurateur Francesco Depoletti (1779-1854) révèle que les sources sont nombreuses et mettent en lumière une approche de la restauration nuancée et basée, dans certains cas, sur des principes de rigueur scientifique et de lisibilité. Les dérestaurations peu précautionneuses que les vases ont subies depuis lors empêchent souvent une étude technique de ces restaurations. Toutefois, les publications et photographies anciennes ainsi que les documents d'archives permettent de retracer les réflexions des savants, des amateurs et des restaurateurs à ce sujet et apportent des éléments fondamentaux à l'étude des vases.

Mots-clés. Histoire de la restauration, archives, dessin, céramique, vases, Grèce, Francesco Depoletti (1779-1854), archéologie, Rome.

\begin{abstract}
The shortcomings of past restoration programms and the inexistence of literature concerning them stem from a dogma that, when challenged by the results of research, runs a strong risk of being discredited. Using the example of the restoration of Greek vases in Italy, especially in Rome, in the 1830s and 1840s, this paper seeks to help debunk this dogma. A dissertation currently being written about the art dealer and restorer Francesco Depoletti (1779-1854) shows the existence of numerous sources and throws light on an approach to restoration that is both nuanced and, in certain cases, based on principles of scientific rigour and legibility. Careless attempts to remove restorations that the vases have undergone since then often prevent a technical analysis of these restorations from being made. However, publications and early photographs, together with archival documents, enable us to follow scientists, connoisseurs and restorers' trains of thought on the subject, and add fundamental data to the study of these vases.

Keywords. Restoration history, archives, drawing, ceramics, vases, Greece, Depoletti (1779-1854), archaeology, Rome.
\end{abstract}

Jusque dans les années 1980, le caractère non scientifique des restaurations du passé était un dogme bien établi. Les interventions que les Penelli réalisèrent sans grande méthode et sans vergogne sur de nombreux vases de la collection Campana ${ }^{1}$ condamnèrent longtemps leurs confrères à l'opprobre. Pourtant, depuis environ quinze ans, des études récentes ont montré que dans la première moitié du XIX ${ }^{\mathrm{e}}$ siècle, à Paris, Naples ou Rome, les restaurations de vases grecs furent souvent élaborées avec un grand raffinement intellectuel et technique : dans de nombreux cas, on mena une réflexion très fine sur les principes de la restauration, qu'il s'agisse de l'étude de l'iconographie ${ }^{2}$ et du style ou de la recherche d'alternatives à la retouche illusionniste, comme le mezzo restauro ${ }^{3}$. Parallèlement, dans le secret des ateliers, on mettait au point des recettes de colles durables ${ }^{4}$, de repeints noirs aussi profonds que le vernis antique, et on débordait d'ingéniosité pour assurer la stabilité d'œuvres incomplètes ${ }^{5}$. On pourra toujours trouver des exemples de restaurations qui s'approchent davantage du bricolage, mais, dans bien des cas, il est évident que cette image de pratiques d'amateur n'est pas adaptée. De plus, les rapports étroits entre restaurateurs et savants interdisent d'accuser uniquement les premiers d'interventions aujourd'hui jugées abusives : les savants s'intéressaient aux restaurations et pouvaient les commander ou les valider.

Pourtant, cette image négative est d'autant plus difficile à détruire que l'archéologie de ces travaux n'est faite que depuis $\mathrm{peu}^{6}$; l'indignité décrétée des résultats a fait longtemps se détourner de l'étude des gestes, des méthodes et des principes de tous ces praticiens sans lesquels les collections les plus prestigieuses des musées européens n'auraient pas eu l'histoire qu'on leur connaît. Il est vrai que les dérestaurations opérées depuis lors dans de nombreux musées rendent la tâche difficile. Néanmoins, l'écriture de cette histoire reste possible : certains vases ont gardé leurs anciennes restaurations que l'on peut observer et analyser jusque dans leur composition chimique. Mais surtout, les publications anciennes, les dessins, documents comptables et correspondances permettent 
de comprendre quels furent les acteurs, les principes et les méthodes de ces restaurations.

C'est ce que cet article souhaite démontrer à travers l'exemple du foyer romain dans les années 1830. À cette période, Naples n'avait plus en effet le monopole de la restauration des vases antiques; à partir de la fin des années 1820, l'exploration archéologique de l'Étrurie méridionale porta au jour des milliers de vases sortis des nécropoles de Vulci, Tarquinia ou Cerveteri. Des fouilles de grande ampleur y furent entreprises ou autorisées par des propriétaires terriens comme Lucien Bonaparte, Agostino Feoli, les frères Candelori ou la famille Campanari. Une fois les vases exhumés, il fallait les nettoyer et les restaurer pour remédier à leur état souvent fragmentaire. Dans les années qui suivirent, on vit donc apparaître dans la littérature savante les noms de Capranesi, Depoletti, Ruspi et d'autres, qui ajoutèrent la restauration à leur profession première de marchand ou d'artiste. Au même moment, Rome abrita la naissance de l'Instituto di Corrispondenza Archeologica, organisme européen qui joua un rôle fondamental ${ }^{7}$; ses membres étaient des archéologues, tels Eduard Gerhard et Emil Braun, des érudits, des collectionneurs, des marchands. La coïncidence de ces activités marchandes et académiques permit qu'elles se nourrissent mutuellement. De plus, la topographie même favorisa une grande porosité de ces milieux. Les ateliers et les boutiques, les pensions pour voyageurs se trouvaient tous à deux pas de la place d'Espagne et du Caffè Greco, fréquenté par toute l'intelligentsia : dans une ambiance d'effervescence autour de l'étude des vases antiques, marchands, restaurateurs, collectionneurs et savants se rencontraient donc assidûment. Les restaurations des années 1830 et 1840 sont ainsi d'autant plus précieuses qu'elles furent effectuées à un moment fondateur de l'étude de la céramique grecque.

\section{Dans le secret des ateliers ?}

Bien des restaurations du passé furent validées par les collectionneurs et les archéologues, et non élaborées à la dérobée au fond d'un atelier. Les correspondances sont de ce point de vue une source d'informations importante : on y lit les choix de restauration envisagés, et s'il n'est pas toujours possible d'identifier les vases, on peut néanmoins comprendre quels étaient les principes adoptés.

L'un des procédés communément utilisés par les restaurateurs et approuvés par les savants était l'analogie : le rapport de ressemblance entre deux motifs iconographiques légitimait, pensait-on, la restitution de l'un d'après le modèle de l'autre. Ainsi, lorsqu'une scène incomplète présentait le même schéma qu'une autre bien conservée, on considérait légitime de compléter la première sur le modèle de la seconde ${ }^{8}$. C'est ce que pensa faire l'archéologue Emil Braun sur un vase incomplet, dont il souhaitait voir compléter le décor par analogie avec un autre vase de sa connaissance : il se félicitait d'en avoir déniché un portant la même iconographie et autorisant une restauration ${ }^{9}$. James Millingen, archéologue et marchand que l'on connaît pour ses prises de positions rigoureuses sur la restauration ${ }^{10}$, y eut aussi recours. Ayant reçu de Rome un beau vase grec qui n'avait pas encore été remonté, il constata que la partie inférieure ne lui appartenait pas ; heureusement, le décor figuré ne portait qu'une lacune restreinte dans la figure de Géryon et la comparaison avec un vase portant la même iconographie pouvait guider la restitution des éléments manquants ${ }^{11}$. Le vase de Millingen est une amphore chalcidienne conservée au British Museum (B 155) ${ }^{12}$ : aujourd'hui dérestaurée, elle montre en effet une lacune dans le bas des ailes de Géryon.

Les archives liées à l'ouverture du Museo Gregoriano Etrusco (1837) livrent un autre exemple de ce regard des savants sur les restaurations : avant que leurs auteurs ne soient payés, ces dernières étaient examinées notamment par l'archéologue Pietro Ercole Visconti qui jugeait de leur conformité avec les attentes du musée $\mathrm{e}^{13}$.

\section{Exactitude comptable}

Outre leurs réalisations, les praticiens ont laissé des factures et des notes précieuses. Dans le cas des documents de la main de Francesco Depoletti (1779-1854) ${ }^{14}$, on remarque que leur degré de précision varie selon le client auquel ils étaient destinés. Pour un particulier comme Pietro Paolo Spagna, pour lequel Depoletti restaura une partie du matériel archéologique provenant des fouilles de Castel Campanile, les factures sont succinctes : la description des pièces concernées est trop générique pour permettre leur identification ; en revanche, celle de l'intervention, bien que brève, permet de distinguer plusieurs types d'opérations. Sur la facture du 8 mars $1832^{15}$, il est intéressant de noter les différentes interventions effectuées : tutto restauro, par opposition au mezzo restauro évoqué plus haut, est une restauration complète dans laquelle tous les éléments sont réintégrés. Les autres vases ont été simplement recollés (incollato) et l'un d'eux portait, suite à son enfouissement, des concrétions dont il a été débarrassé (levato il tartaro duro). Cette simple facture donne donc un aperçu intéressant des méthodes de restauration, car elle prouve qu'il n'existait pas que la seule retouche illusionniste ; dans le cas des seize vases ici mentionnés, elle n'a été mise en œuvre que deux fois, ce qui égratigne sérieusement la thèse de la restauration comme un maquillage effectué sans discernement. Ce document donne aussi une idée des prix demandés : pour la restauration complète du vaso a olla (probablement un stamnos), trente-cinq $s c u d i^{16}$; pour le recollage de deux coupes et d'un autre vase probablement peu fragmentaire, un scudo et demi ; pour un vase brisé en de nombreux morceaux, deux scudi. On remarque un écart important entre les prix : un demi scudo pour un recollage simple, contre trentecinq pour une restauration complète. Or on sait que le salaire journalier d'un ouvrier sur un chantier de fouilles s'élevait à un quart de $s c u d o^{17}$, et qu'un fiacre pour aller du Vatican à la via dei Condotti coûtait le même prix ${ }^{18}$. La restauration avait donc un coût élevé puisque celle du stamnos revenait à cent 
quarante fois le salaire journalier de l'ouvrier qui l'avait exhumé.

Les factures établies pour le Museo Gregoriano Etrusco sont plus détaillées, et l'on regrette qu'elles n'aient pas toutes été conservées ${ }^{19}$ : la description des opérations est aussi précise, et dans la majorité des cas, les vases peuvent être identifiés. Les opérations sont de divers types : les factures ne font pas apparaître les mentions tutto restauro ou mezzo restauro mais listent plusieurs degrés d'interventions. Les vases sont souvent nettoyés, puis retouchés : "pulito e ripreso il colore »; certains sont dits restaurés, sans plus de mention : «Campana [...], restaurata». L'un des cas les plus intéressants est celui d'un grand cratère apulien déjà restauré et sur lequel Depoletti dut intervenir ; il décrivit son travail ainsi : "Vaso grandissimo a mascheroni di Ruvo, pulito, levati li stucchi vecchi, ristuccato e dato il colore $e^{20}$. » Le rapprochement avec un dessin réalisé dans l'atelier de Depoletti21 ${ }^{21}$ permet d'identifier ce vase : il s'agit d'un cratère apulien à figures rouges attribué au Groupe de Varrese et datant des années 360-340 avant J.-C. ${ }^{22}$ Il appartenait au noyau ancien de la Bibliothèque Vaticane et fut transféré à Paris suite au traité de Tolentino (19 février 1797) ; probablement restauré à Paris ${ }^{23}$, il fut exposé au Musée Napoléon de 1800 à 1815, puis restitué au Vatican ${ }^{24}$. Transféré de la Bibliothèque Vaticane au Museo Gregoriano Etrusco, il fut de nouveau restauré en 1837 par Francesco Depoletti. L'examen et les analyses des matériaux de restauration effectués par le Centre de Recherche et de Restauration des Musées de France sous la conduite de Brigitte Bourgeois confirment cette double intervention ${ }^{25}$.

\section{Dessins et relevés}

Une autre source documentaire importante est constituée par les dessins. Pour l'étude du foyer romain, le fonds le plus riche est le Gerhard'scher Apparat : Eduard Gerhard (17951867), qui fut à l'origine de la fondation de l'Instituto di Corrispondenza Archeologica, se tenait très au courant de toutes les découvertes réalisées en Étrurie méridionale et déplorait la dispersion du matériel archéologique. Pour y remédier et pour renouveler la science archéologique par une étude plus systématique, il constitua un Apparat composé, entre autres, de plusieurs milliers de dessins exécutés en majorité à Rome dans les années 1830 et 1840 ; ils sont aujourd'hui un outil précieux pour l'histoire des fouilles, de la restauration et des collections ${ }^{26}$. Ils portent en effet plusieurs types d'annotations indiquant le lieu de provenance, l'état du vase et le nom du propriétaire, du marchand ou du restaurateur chez qui l'œuvre était dessinée : c'est ainsi que l'on peut identifier le cratère à volutes du Vatican restauré par Depoletti. Le bon état de conservation est en général indiqué par les mentions «Sano» ou «Completo». Outre ces annotations, nombre de dessins présentent l'indication graphique des restaurations. Selon le dessinateur, elles peuvent prendre plusieurs formes : souvent, une ligne continue ou pointillée définit la lacune avec parfois une mention telle "mancante»; la partie manquante tantôt est laissée vide, tantôt montre la restauration effectuée. Il existe même des dessins différents pour un même vase : la coupe attique à figures rouges attribuée au Peintre de Brygos et conservée au Palazzo Schifanoia à Ferrare ${ }^{27}$ est ainsi représentée deux fois. Sur l'un des dessins (fig. 1) dans le décor du tondo et de la face B, les lacunes sont laissées blanches et sont entourées d'une ligne pointillée. L'autre dessin (fig. 2) ne laisse rien deviner de l'état du vase : toutes les lacunes sont restituées, et aucune ligne ou mention ne vient préciser ce qui est manquant. Ces deux relevés documentent donc deux états différents du vase.

\section{Des publications sans ambiguité}

Dans les publications des années 1830 et 1840, on remarque que l'état de conservation des vases est souvent précisé dans le texte ou sur les planches, ainsi que le recommandait au début du siècle Aubin-Louis Millin²8. Le premier cas est, convenons-en, assez rare : Lucien Bonaparte donna à plusieurs reprises dans son Museum étrusque des indications sur les choix de restaurations opérés pour tel ou tel vase ; à propos d'une hydrie attique à figures rouges aujourd'hui conservée à Munich ${ }^{29}$, il écrivit : "Ce vase précieux a été trouvé en fragments et à différents jours d'intervalle; heureusement les figures et les inscriptions sont demeurées entières excepté quelques éclats dans le corps d'un athlète ; tous ces fragments ont été réunis en sorte cependant qu'on peut les compter tous ; et cette demi-restauration sans ajouter une seule ligne de dessin nous paraît la seule que l'on devrait se permettre dans les monuments de l'antiquité ${ }^{30}$. » On a là un exemple de ses principes stricts en matière de restauration selon lesquels le vase devait être complété dans sa forme, mais non dans son décor ${ }^{31}$. Lucien Bonaparte n'était pas toujours aussi précis mais veillait à indiquer l'état du vase par les termes « intact », « complet » et « incomplet ${ }^{32}$ ». De même Secondiano Campanari, dans son catalogue de la collection Feoli, précisa l'état de conservation d'une amphore du Peintre de Cléophradès et justifia le choix d'une restauration visible par l'étendue des lacunes et le respect de la matière antique ${ }^{33}$.

Quand l'ouvrage comporte des planches, ce sont elles le plus souvent qui donnent ce type d'indication. Si l'on compare dessins préparatoires et illustrations définitives, l'ouvrage d'Eduard Gerhard Auserlesene Griechische Vasenbilder hauptsächlich Etruskischen Fundorts n'indique pas toujours les lacunes : quand elles ne concernent qu'un motif sans surprise telle une colonnette soutenant un bassin, ou qu'elles sont très restreintes, le vase est publié comme s'il était intact ${ }^{34}$. En revanche, les lacunes importantes sont souvent indiquées, même approximativement, par des lignes de points ou par des zones laissées blanches dans lesquelles un trait vient suggérer les éléments disparus. Une enquête plus approfondie sur l'histoire des vases concernés permet d'établir que ces deux modes graphiques correspondent à deux types de restauration : lorsque, sur la planche gravée, le décor semble complet et comporte l'indication de zones cernées par des points, il faut comprendre 


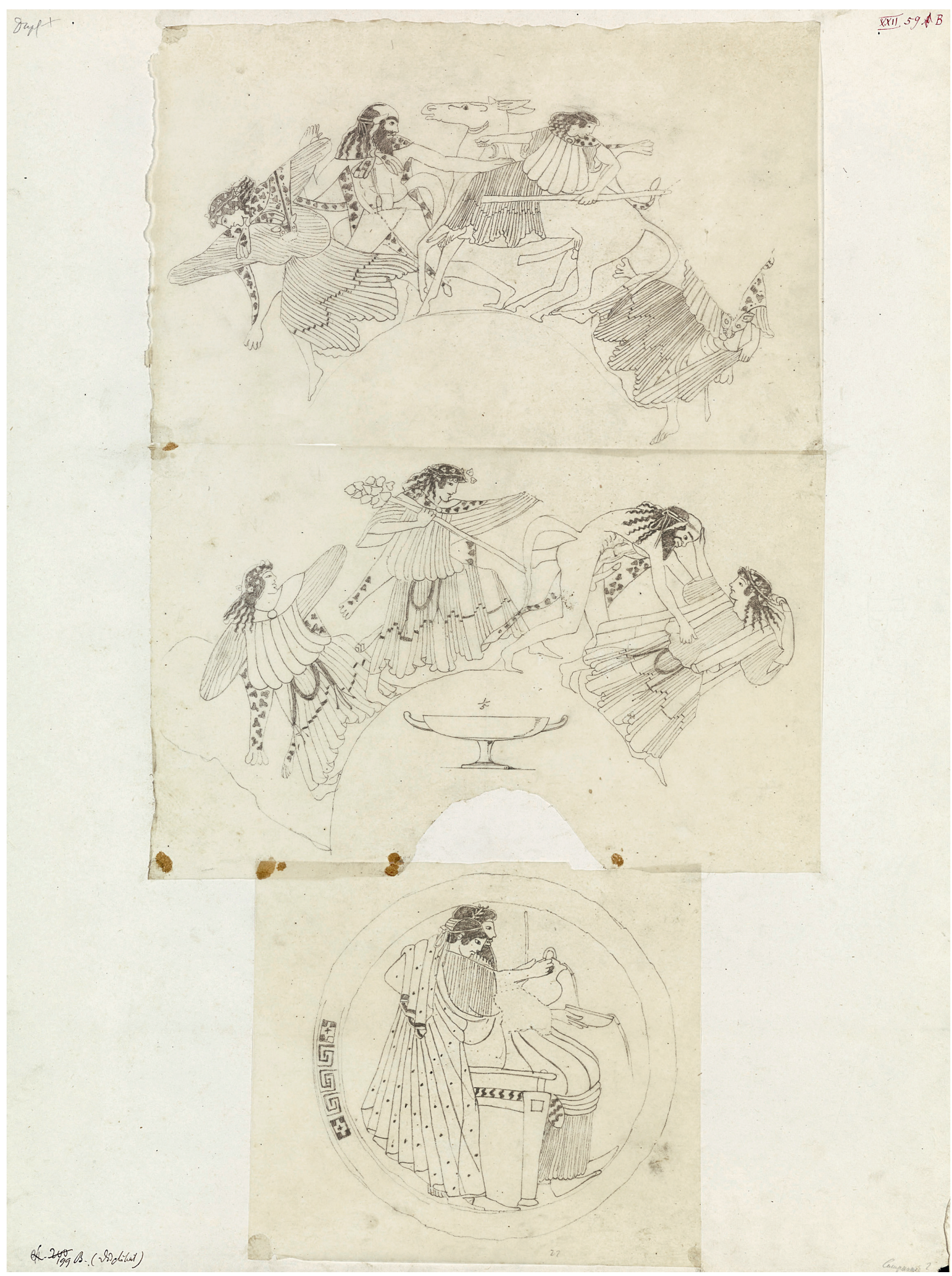

Fig. 1. Dessins d'une coupe attique à figures rouges (Ferrare, Palazzo Schifanoia, 277) avant restauration. Gerh. App. XXII, 59b. ๑ Antikensammlung, Staatliche Museen zu Berlin. Foto J. Laurentius. 


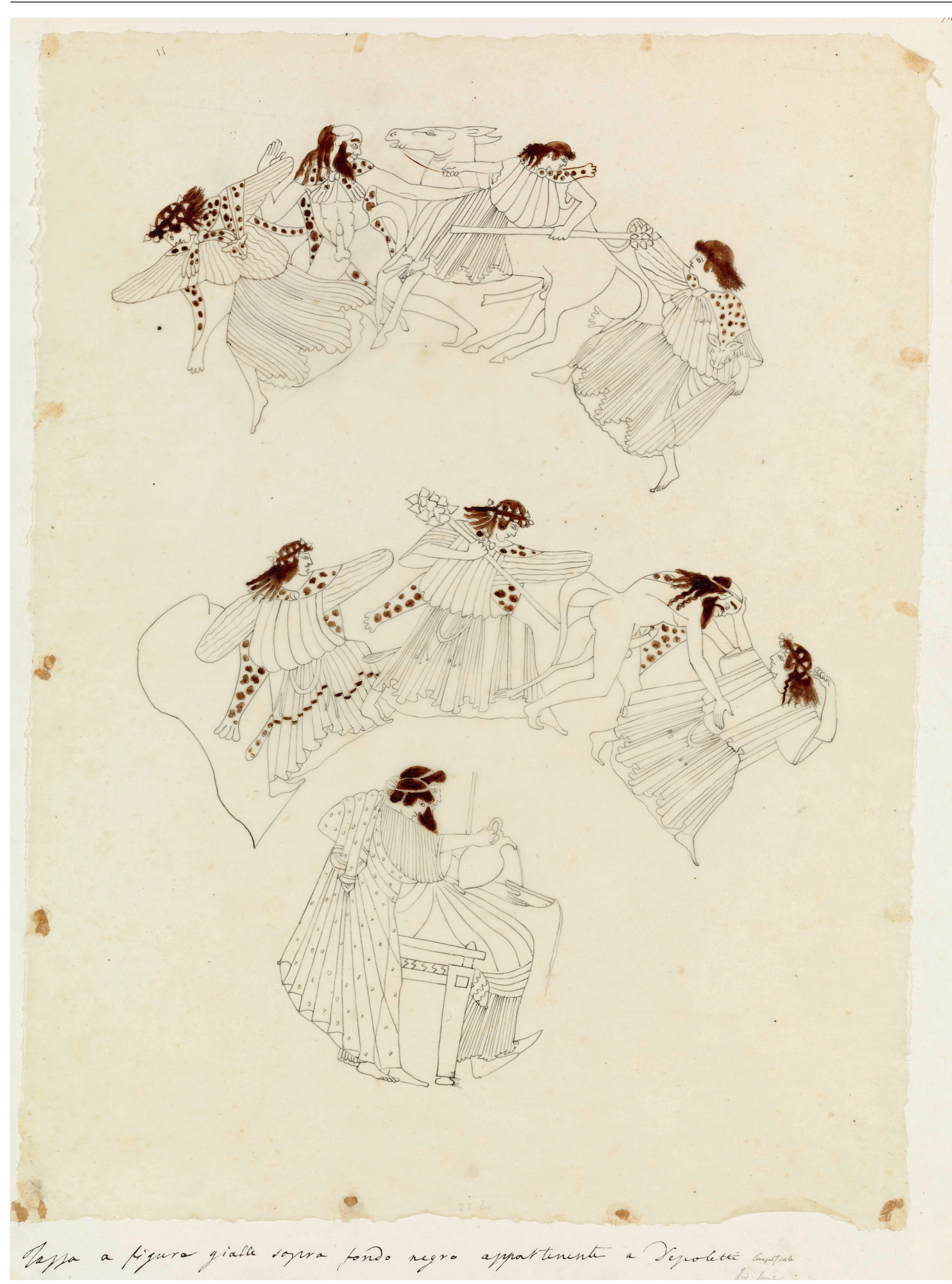

Fig. 2. Dessins d'une coupe attique à figures rouges (Ferrare, Palazzo Schifanoia, 277) après restauration. Gerh. App. XXII, 59a. (c) Antikensammlung, Staatliche Museen zu Berlin. Foto J. Laurentius. 

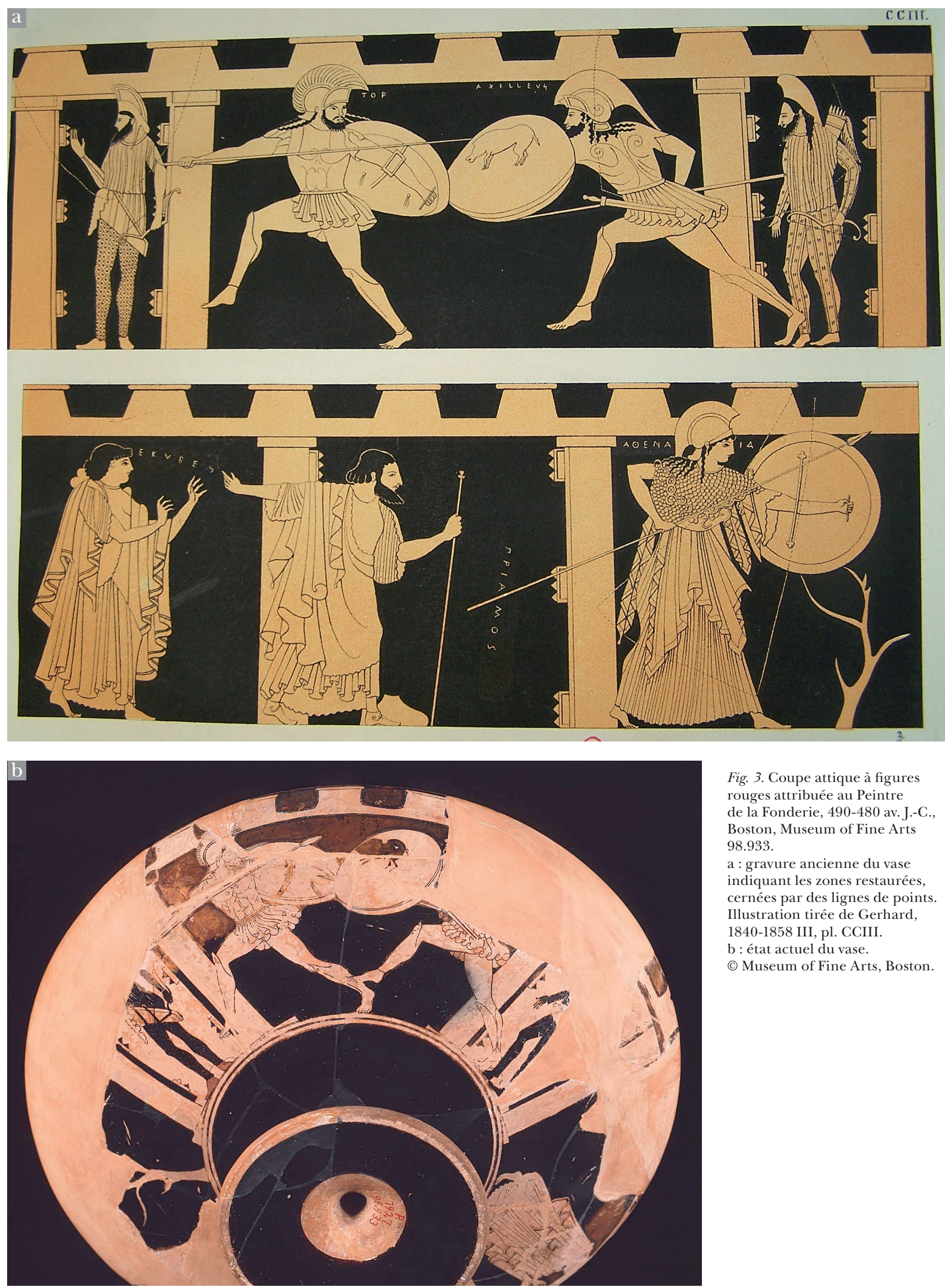

Fig. 3. Coupe attique à figures rouges attribuée au Peintre de la Fonderie, 490-480 av. J.-C., Boston, Museum of Fine Arts 98.933.

a : gravure ancienne du vase indiquant les zones restaurées, cernées par des lignes de points. Illustration tirée de Gerhard, 1840-1858 III, pl. CCIII.

b : état actuel du vase.

(c) Museum of Fine Arts, Boston. 


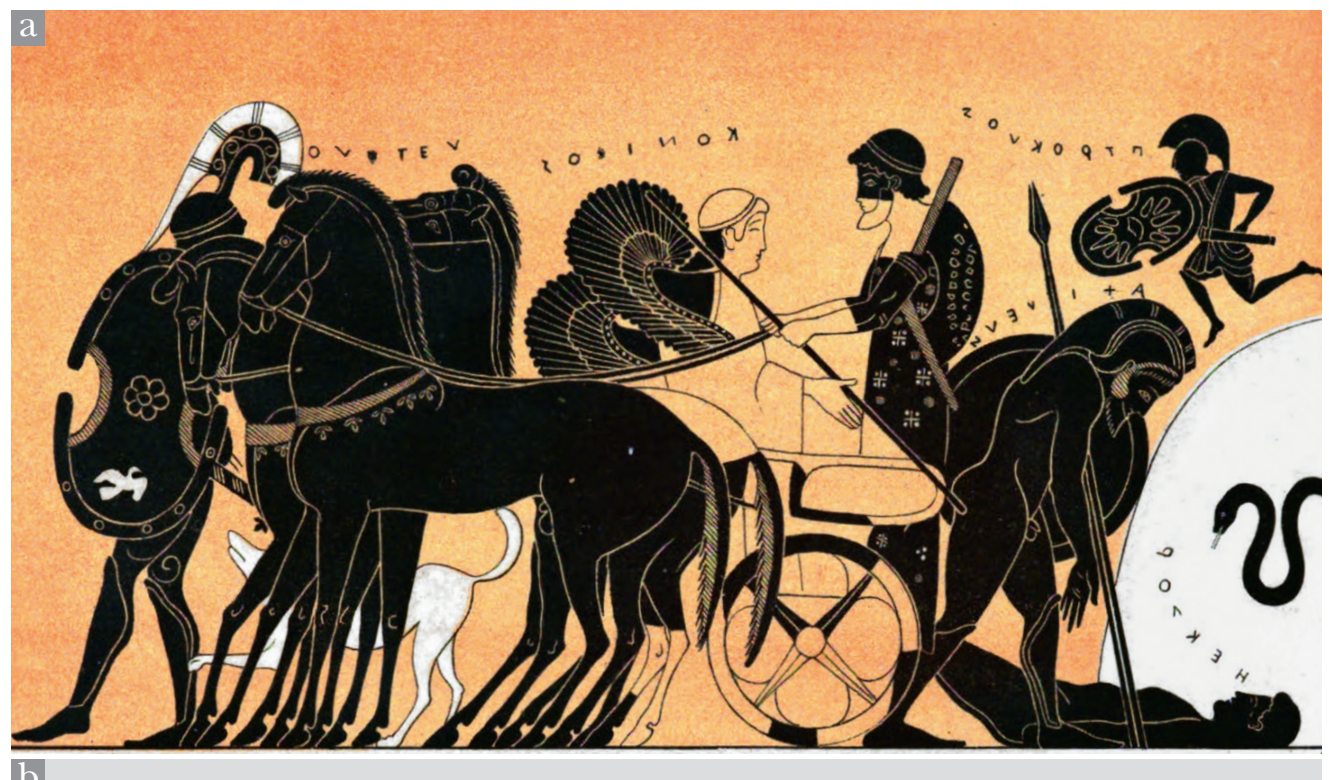

Fig. 4. Amphore attique à figures noires attribuée au Peintre de Priam, 520-510 av. J.-C., Londres, British Museum 1899,0721.3 a : gravure ancienne du vase indiquant les lacunes. Illustration tirée de Gerhard, 1840-1858 III,

pl. CXCIX.

b : état actuel du vase.

(c) The Trustees of the British Museum.

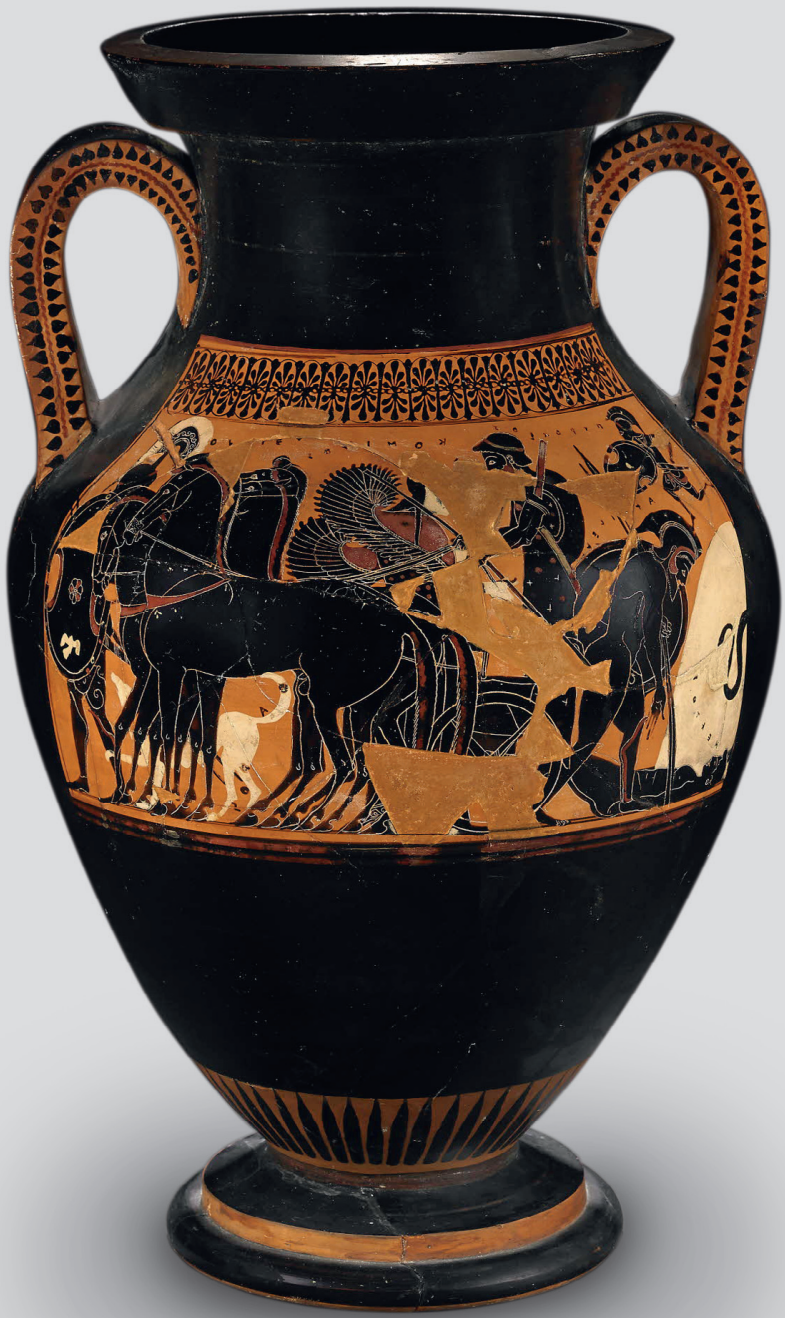


que sur le vase, les lacunes avait bien été réintégrées (fig. 3) Quand la lacune est laissée blanche sur la planche, il s'avère qu'elle avait été laissée telle quelle sur le vase. Les motifs restitués par une ligne sont une simple proposition de Gerhard. (fig. 4).

Lettres, factures, dessins, gravures et textes prouvent donc que la restauration n'était pas toujours, comme on se complaît à l'imaginer, l'activité secrète de semi-faussaires trahissant l'antique pour en tirer le maximum d'espèces sonnantes et trébuchantes. Les interventions abusives ne manquèrent pas, mais les sources démontrent qu'existaient aussi des pratiques élaborées, réfléchies et respectueuses de la matière et de l'image de l'œuvre. L'enquête sur ces restaurations du passé est donc fondamentale pour éviter de célébrer naïvement la modernité et de "produire une histoire qui se rédui[se] à une ratification, sinon à une glorification du présent ${ }^{35}$ ». Elle est également très profitable d'un point de vue épistémologique. On rend hommage à juste titre aux savants qui passèrent de l'antiquarisme à l'archéologie, mais les opposer de manière dichotomique aux marchands-restaurateurs n'est pas, pour le milieu romain des années 1830 et 1840, pertinent. Patiemment, et parfois modestement, ces hommes de l'ombre redonnaient forme et lisibilité aux objets sur lesquels s'édifia cette science nouvelle; ils permettaient qu'on les dessine, y compris en notant les lacunes et les restaurations qui diminuaient leur valeur commerciale. Sans surévaluer leur rôle ni dissimuler les aspects aujourd'hui critiquables de leurs pratiques, il serait donc judicieux de leur reconnaître la place qu'ils occupèrent dans la transmission des vases de l'Antiquité à nos jours, et dans un pan essentiel de l'histoire culturelle de l'Europe.
Notes

1. Bourgeois, 1989. Nadalini, 1992.

Nadalini, 1993. Nadalini, 2013.

2. Bourgeois, 2010b.

3 . Le mezzo restauro repose sur la distance entre le spectateur et le vase : à quelques pas, ce dernier semble complet et rien dans son aspect ne vient perturber le regard, mais de près, les lacunes, qu'on aura simplement mises au ton, se révèlent sans ambiguïté. Cette méthode aurait été élaborée à Naples dans les années 1820. À ce sujet, voir Milanese, 2010, Chazalon, 2010.

4. Milanese, 1996-1997.

5. Voir par exemple Merlin, 2010, p. 83, fig. 1. Sannibale, Santamaria, Morresi, 2013 , fig. 73 .

6. Je renvoie en premier lieu aux travaux de Brigitte Bourgeois (Bourgeois 2010a, b et Bourgeois, Denoyelle 2013).

7. Schnapp, 1993, p. 371-380.

8. Hamilton Gray, 1840, p. 314-316.

9. Lettre d'E. Braun à E. Gerhard, Rome 19 février 1835, Rome DAI.

Costantini, 1998, p. 252.

10. Milanese, 2007. Bourgeois 2010a. Milanese, 2010.

11. Lettre de J. Millingen à E. Braun, Rome 27 février 1836, Rome DAI.

12. Rumpf, 1927, pl. XIII à XV. Ces planches montrent le vase encore restauré : les lacunes sont réintégrées, mais les cassures visibles. Il est difficile de se prononcer sur la date de cet état, et d'affirmer qu'il corresponde à la restauration voulue par Millingen.

13. Archivio di Stato di Roma, C. II, tit. IV, b. 245 , fasc. 2570.

14. Bernard, 2008. Bernard, 2013

15. Archives Spagna. Rome, Museo di Roma, fol. 74

16. Le scudo était la monnaie des États pontificaux ; il peut se traduire par écu, mais pour éviter la confusion avec la monnaie française, le terme est généralement laissé en italien.
17. Archives Spagna. Rome, Museo di Roma, fol. 35.

18. ASR, C. II, tit. IV, b. 245 , fasc. 2570.

19. Archivio di Stato, Roma. C. II, tit. IV., b. 245. Je remercie le Dr. Maurizio Sannibale, conservateur au Museo Gregoriano Etrusco, qui s'apprête à publier ces archives et qui est depuis plusieurs années un interlocuteur bienveillant. Voir G.Pinza, B. Nogara, Documenti relativi alla formazione ed alle raccolte principali del Museo Gregoriano Etrusco, edizione a cura di M. Sannibale, à paraître.

20. «Très grand vase à mascarons de Ruvo, nettoyé, enlevé les vieux mastics, remastiqué et donné la couleur. »

(Ma traduction). Archivio di Stato, Roma.

C.II, tit. IV., b. 245, fasc. 2570.

21. Gerh. App. XVII, 16 (Berlin,

Antikensammlung).

22. Museo Gregoriano Etrusco 17163.

Masci 2008, p. 390, n 136.

23. Matz, 2008. Bourgeois, 2010 b.

24. Denoyelle, Lissarrague, 2003.

25. Sannibale, Santamaria, Morresi, 2013.

26. Le fonds est conservé à l'Altes Museum à Berlin, et au Deutsches Archäologisches Institut à Rome. Je remercie le Dr. Ursula Kästner et le Dr. Thomas Fröhlich de m'avoir permis de consulter ces dessins.

27. Ferrare, Palazzo Schifanoia, 277.

28. Voir l'article de B. Bourgeois dans ce volume.

29. Hydrie attique à figures rouges,

Munich, Antikensammlung 2420

30. Bonaparte, 1829, p. 34.

31. Pour un autre exemple, voir Bonaparte, 1829, p. 75 : "Ces deux coupes étant du plus beau travail nous les avons fait rassembler par l'habile restaurateur romain M. Depoletti qui avec un art parfait a rempli les vides sans toucher en rien au dessin, de sorte que l'on peut compter le nombre de fragments de chaque coupe, et ce remplissage moderne se voit au premier coup d'œil sans pouvoir se confondre en rien avec l'antique. " Les coupes sont conservées au Louvre (G 6) et au British Museum (E41).

32. Bonaparte, 1829.

33. Campanari, 1837, p. 198. Sur la restauration de ce vase (Würzburg L 508), voir Bernard, 2013.

34. Gerhard, 1840-1858, IV, pl. CCLXXII : le vase non localisé semble intact, mais les dessins du Gerhard'scher Apparat (XVI, 9.3 et XXI, 2.1) laissent voir de très petites lacunes. 35. Gould, 2003, p. 160.

\section{Bibliographie}

Bernard M.-A., 2008, « Francesco Depoletti (1779-1854), artiste et restaurateur de vases antiques à Rome vers 1825-1854 ", Technè, $\mathrm{n}^{\circ} 27-28,2008$, p. 79-84.

Bernard M.-A., 2013, « Francesco Depoletti (1779-1854), un homme de réseaux entre collectionnisme et restauration ", dans Bourgeois B. et Denoyelle M. (dir.), L'Europe du vase antique.

Collectionneurs, savants, restaurateurs aux XVIII ${ }^{e}$ et XIX ${ }^{e}$ siècles., Paris, Institut National d'Histoire de l'Art - Centre de Recherche et de Restauration des Musées de France - Presses Universitaires de Rennes, p. 203-220.

Bonaparte L., 1829, Museum étrusque de Lucien Bonaparte prince de Canino. Fouilles de 1828 à 1829. Vases peints avec inscriptions. C. Tosoni, Viterbe.

Bourgeois B., 1989, « La relecture d'une ouvre anonyme : le dinos du "Peintre de la Gorgone" au musée du Louvre ", dans Anonymat et signature, La Documentation française, Paris, p. 95-105.

Bourgeois B., 2010 a, « La fabrique du vase grec. Connaître et restaurer l'Antique dans l'Europe des Lumières - Genèse d'un projet ", dans Bourgeois B. (dir.), 
"Une perfection dangereuse ». La restauration des vases grecs de Naples à Paris, XVIII'-XIX ${ }^{e}$ siècles, Technè, $\mathrm{n}^{\circ} 32$, p. 5-10.

Bourgeois B., 2010 b, « Le laboratoire de l'antique. Luigi Brocchi, restaurateur de vases au musée Napoléon. ", dans Bourgeois B. (dir.), «Une perfection dangereuse ". La restauration des vases grecs de Naples à Paris, XVIII ${ }^{e}$-XIX ${ }^{e}$ siècles, Technè, no 32, p. 60-70.

Bourgeois B., Denoyelle M. (dir.), L'Europe du vase antique. Collectionneurs, savants, restaurateurs aux XVIII et XIX ${ }^{e}$ siècles. Paris, Institut National d'Histoire de l'Art - Centre de Recherche et de Restauration des Musées de France Presses Universitaires de Rennes.

Campanari S., 1837, Antichi vasi dipinti della collezione Feoli, Rome.

Chazalon L., 2010 « Les vases attiques à figures noires restaurés dans le laboratoire de Raffaele Gargiulo à Naples. Étude pratique d'un regard d'époque », dans Bourgeois B. (dir.) "Une perfection dangereuse".

$L$ a restauration des vases grecs de Naples à Paris, XVIII ${ }^{e}-X I X^{e}$ siècles, Technè, $\mathrm{n}^{\circ} 32$, p. 31-37.

Costantini A., 1998, Roma nell'età della restaurazione : un aspetto della ricerca archeologica. La collezione di vasi attici di Luciano e Alexandrine Bonaparte, riprodotta nei disegni del «Gerhard'scher apparat», Roma, Accademia nazionale dei Lincei, 1998 (vol. 10).

Denoyelle M., Lissarrague F., 2003, « Destins de vases ", dans Le Vase grec et ses destins, Biering \& Brinkmann, München, p. $215-227$

Gerhard E., 1840-1858, Auserlesene griechische Vasenbilder : hauptsächlich etruskischen Fundorts, G. Reimer, Berlin.
Gould S. J., 2003, Le renard et le hérisson. Pour réconcilier les sciences et les humanités, nouvelle édition, Le Seuil, Paris.

Hamilton Gray E. C., 1840, Tour to the sepulchres of Etruria, in 1839, J. Hatchard and Son, Londres, $578 \mathrm{p}$.

Masci M. E., 2008, Picturae Etruscorum in Vasculis. La raccolta Vaticana e il collezionismo di vasi antichi nel primo Settecento, L'Erma di Bretschneider, Rome.

Matz F., 2008, «Jean-Jacques Lagrenée (1739-1821) et le vase grec ", dans Bourgeois B. (dir.), La restauration des cuuves d'art. Éléments d'une histoire oubliée, XVIII ${ }^{e}$-XIX ${ }^{e}$ siècles, Technè, $\mathrm{n}^{\circ}$ 27-28, p. $47-52$.

Merlin C., 2010, « Conserver les traces. Comment traiter d'anciennes restaurations? ", dans Bourgeois B. (dir.), "Une perfection dangereuse ». La restauration des vases grecs de Naples à Paris, XVIII $-X I X^{e}$ siècles, Technè, $\mathrm{n}^{\circ} 32$, p. $81-90$.

Milanese A., 1996-1997, « Il Museo Reale di Napoli al tempo di G.Bonaparte e di G.Murat ", Rivista dell' Instituto Nazionale di Archeologia e Storia dell' Arte, XIX-XX, p. $403-404$.

Milanese A., 2007, " "Pour ne pas choquer l'œil". Raffaele Gargiulo e il restauro di vasi antichi nel real Museo di Napoli: opzioni di metodo e oscillazioni di gusto tra 1810 e 1840 ", dans Gli Uomini e le cose. 1. Figure di restauratori e casi di restauro in Italia tra XVIII e XX secolo, Clio Press, Naples, p. 82-103.

Milanese A., 2010, «De la "perfection dangereuse" et plus encore. La restauration des vases grecs à Naples au début du XIX ${ }^{\mathrm{e}}$ siècle, entre histoire du goût et marché de l'art », dans
Bourgeois B. (dir.), "Une perfection dangereuse ". La restauration des vases grecs de Naples à Paris, XVIII ${ }^{e}-X X^{e}$ siècles, Technè, $\mathrm{n}^{\circ} 32$, p. 18-30.

Nadalini G., 1992, «Le musée Campana : origine et formation des collections. L'organisation du musée et le problème de la restauration ", dans L'Anticomanie : la collection d'antiquités aux $18^{e}$ et $19^{e}$ siècles, Éd. de l'École des hautes études en sciences sociales, Paris.

Nadalini G., 1993, «Il Ripristino di un grande cratere attribuito ad Eufronio », CeramicAntica, $\mathrm{n}^{\circ} 26,1993$, p. 28-34.

Nadalini G., 2013, « Enrico Penelli : la trajectoire d'un restaurateur de vases étrusques du XIX ${ }^{\mathrm{e}}$ siècle ", dans Bourgeois B., Denoyelle M. (dir.), L'Europe du vase antique. Collectionneurs, savants, restaurateurs aux XVIII ${ }^{e}$ et $X I X^{e}$ siècles., Paris, Institut National d'Histoire de l'Art - Centre de Recherche et de Restauration des Musées de France - Presses Universitaires de Rennes, p. 185-199.

Rumpf A., 1927, Chalkidischen Vasen, Walter de Gruyter, Berlin, Leipzig.

Sannibale M., Santamaria U., Morresi F., 2013, « Les vases du Vatican autrefois au musée Napoléon et leurs restaurations ", dans Bourgeois B., Denoyelle M. (dir.), L'Europe du vase antique. Collectionneurs, savants, restaurateurs aux $\mathrm{XVIII}^{e}$ et XIX ${ }^{e}$ siècles, Paris, Institut National d'Histoire de l'Art - Centre de Recherche et de Restauration des Musées de FrancePresses Universitaires de Rennes, p. 121-138.

Schnapp A., 1993, La conquête du passé : Aux origines de l'archéologie, Carré, Paris, 384 p. 